\title{
Competências Essenciais ao Terapeuta Cognitivo-Comportamental
}

\author{
Isabela Lamante Scotton ${ }^{1}$ \\ Janaina Bianca Barletta ${ }^{1}$ \\ Carmem Beatriz. Neufeld \\ ${ }^{1}$ Universidade de São Paulo, Ribeirão Preto, São Paulo, Brasil
}

\begin{abstract}
Resumo
O presente estudo tem como objetivo realizar uma revisão integrativa da literatura latino-americana acerca de quais são as competências essenciais para um terapeuta cognitivo-comportamental, visto que as pesquisas na região são escassas e o desenvolvimento de competências profissionais fortalece a prática clínica. Foi realizado um levantamento bibliográfico por meio do mapeamento do que se tem publicado nas bases de dados PsycINFO, LILACS, SciELO e PePSIC. Foram incluídos na síntese qualitativa final oito artigos. As competências essenciais foram categorizadas em competências analíticas, sociais, instrumentais e de autoconhecimento e autorreflexão. Entre os principais achados estão as competências essenciais descritas nos artigos. Dentre estas, considera-se que a maior contribuição foi em relação às competências sociais, como uma competência inespecífica ou geral, necessária a todas as psicoterapias independentemente da orientação teórica de base. O presente estudo visou contribuir com a temática, visto que os estudos em âmbito nacional e latino-americano são escassos.
\end{abstract}

Palavras-chave: competência profissional, terapeutas, terapia cognitivo-comportamental

Essential Skills for the Cognitive-Behavioral Therapist

\begin{abstract}
There is a shortage of studies on essential skills for a cognitive-behavioral therapist in Latin America, so this study aims to conduct an integrative review of the existing literature in the region, since the development of professional skills is essential to strengthen clinical practice. A bibliographic survey was performed by mapping what has been published in the PsycINFO, LILACS, Scielo, and Pepsic databases. Eight articles were included in the final qualitative synthesis. Core competencies were categorized into analytical, social, instrumental, self-knowledge, and self-reflection skills. The main findings comprise the essential skills described in the articles. Among these, it is considered that the greatest contribution was in relation to social competencies, as a non-specific or general competence, necessary to all psychotherapies regardless of the basic theoretical orientation.

Keywords: professional competence; therapists; cognitive-behavioral therapy
\end{abstract}

Habilidades Esenciales para el Terapeuta Cognitivo Conductual

\begin{abstract}
Resumen
Este estudio tuvo como objetivo realizar una revisión integradora de la literatura latinoamericana sobre cuáles son las competencias esenciales para un terapeuta cognitivo conductual, dado que la investigación en la región es insuficiente y el desarrollo de habilidades profesionales fortalece la práctica clínica. Se realizó una encuesta bibliográfica mediante el mapeo de lo publicado en las bases de datos PsycINFO, LILACS, Scielo y Pepsic. Se incluyeron ocho artículos en la síntesis cualitativa final. Las competencias básicas se caracterizaron en habilidades analíticas, sociales, instrumentales, de autoconocimiento y autorreflexión. En los principales hallazgos se encuentran las habilidades esenciales descritas en los artículos. Entre estos, se considera que la mayor contribución fue con relación a las competencias sociales, como una competencia no específica o general, necesaria para todas las psicoterapias, independientemente de la orientación teórica básica. Este estudio tuvo como objetivo contribuir a la temática, ya que los estudios a nivel nacional y latinoamericano son escasos.

Palabras clave: competencia profesional; terapeutas; terapia de conducta cognitiva
\end{abstract}

O movimento da Psicologia em direção à prática baseada em evidências tem crescido consideravelmente nas últimas décadas (Melnik, Meyer, \& Sampaio, 2019). Entende-se que o uso de evidências na prática clínica aumenta a qualidade da intervenção disponibilizada, bem como fortalece as competências profissionais necessárias para uma atuação ética e responsável (Allan, McLuckie, \& Hoffecker, 2016; Milne, 2018; Newman \& Kaplan, 2016). Baseado nesse raciocínio, diversos sistemas de saúde de países ao redor do mundo buscaram implantar a prática baseada em evidências. Nos Estados Unidos, por exemplo, a Psicologia baseada em evidência foi adotada como política de saúde, após um relatório da Força Tarefa da American Psychological Association (APA, 2005) ressaltando os custos e benefícios dessa prática. No Reino Unido, o governo britânico lançou uma iniciativa em larga escala denominado Programa de Acesso às Terapias Psicológicas (Improving Access to Psychological Therapies - IAPT), com dois intuitos: aumentar o acesso da população à psicoterapia e 
identificar terapias que tivessem bases em evidências (Barletta \& Neufeld, 2020).

Para tanto, houve um esforço pelo desenvolvimento de diretrizes para as melhores práticas (APA, 2005). Por exemplo, Roth e Pilling (2007), em parceria com a IAPT, publicaram uma diretriz delineando as principais competências necessárias para oferecer a Terapia Cognitivo-Comportamental (TCC) eficaz, agrupadas em cinco domínios: competências terapêuticas genéricas, competências básicas em TCC, técnicas específicas de TCC, competências específicas de problemas e metacompetências. A partir desse ponto, nos últimos 12 anos, uma série de diretrizes para terapias baseadas em competências tem sido publicadas e cada vez mais refinadas (Andersson, 2016; Barletta \& Neufeld, 2020; Price, Callahan, \& Cox, 2017).

A TCC, por sua vez, é baseada em evidências e com altos índices de validação científica, demonstrando sua eficácia em diferentes transtornos psiquiátricos (Rangé, Pavan-Cândido, \& Neufeld, 2017), portanto têm chamado atenção nas últimas décadas. Por outro lado, a literatura vem apontando uma lacuna entre pesquisas testadas empiricamente e a prática clínica vigente em TCC (Reis \& Barbosa, 2018; Wenzel, 2018), o que requer extrema atenção sobre sua aplicabilidade. Nesse sentido, pesquisadores têm se preocupado com o processo de formação e treinamento de terapeutas, a fim de garantir condições para o desenvolvimento de competências profissionais adequadas (Honorato \& Barletta, 2016; Muse \& McManus, 2016; Souza \& Padovani, 2015). Essa preocupação, entretanto, é ainda incipiente, notadamente no âmbito nacional e latino-americano (Barletta \& Neufeld, 2020).

Ao se falar de competências, a literatura tem apontado uma definição amplamente utilizada ao se falar em competência profissional como um todo. Tal conceito se refere a uma combinação sinérgica de conhecimentos, habilidades e atitudes (CHAs), expressa pelo desempenho profissional em determinado contexto e que podem ser adquiridas por meio do processo de aprendizagem (Brandão \& Bahry, 2014; Fung \& Lo, 2017). Para Carbone, Tonet, Bruno, Silva e Izidio (2016), o conhecimento diz respeito ao "saber por que fazer", o que envolve a compreensão de princípios teóricos e seus propósitos. A habilidade está relacionada à capacidade de fazer uso produtivo do conhecimento que possui, promovendo a articulação entre os conhecimentos, referindo essa dimensão ao "saber como fazer".

Por fim, a atitude, por sua vez, refere-se aos aspectos sociais e afetivos relacionados ao trabalho, ligado ao sentimento ou grau de aceitação e rejeição da pessoa em relação aos outros. Os autores afirmam que o efeito da atitude é ampliar a reação positiva ou negativa de uma pessoa, sua predisposição em relação a uma ação específica. Por analogia, referem essa dimensão como o "querer fazer", sendo apontada como um fator de intencionalidade (Carbone, Tonet, Bruno, Silva, \& Izidio, 2016).

Dentre os estudos de competência clínica, há aqueles que diferenciam duas classes de competências: técnicas e interpessoais (Pascual-Leone, Andreescu, \& Yeryomenko, 2015)psychological or pedagogy studies are more effective and competent than other graduates. Research sample and methods: The sample included 235 psychotherapist-patient dyads, where psychotherapists where students of the Integrative Psychotherapy School. Before they started training, they had graduated various MA studies: in psychology, pedagogy, theology, medicine, nursing, languages, law, other humanities or natural sciences. The subject of rating was a therapeutic session. After the session finished, the patient completed a detailed rating scale concerning the psychotherapist and the session. The psychotherapist competences were rated according to the rating scale regarding personal and technical competences, based on the observable criteria. Non-parametric methods were used to calculate the results. Results: Psychologists, doctors and pedagogy specialists were equally effective and competent as other professions. Thus., no empirical justification has been discovered to privilege the psychologists, doctors and pedagogy specialists when providing access to professional psychotherapist training. (PsycINFO Database Record (c. Outros estudos sugerem a diferenciação entre competências gerais, isto é, aquelas que todo terapeuta precisa ter independentemente da abordagem, e competências específicas, que são decorrentes do modo de trabalho de cada abordagem teórica (Kaplowitz, Safran, \& Muran, 2011).

Em termos de TCC, estudos vêm propondo algumas competências essenciais. Por exemplo, Honorato e Barletta (2016) destacam quatro classes de competências essenciais: teóricas, técnicas, interpessoais e de autoconhecimento do terapeuta. De acordo com as autoras, tais classes de competências devem ser entendidas como aspectos interligados para a prática clínica. Essa inter-relação entre as competências de diferentes classificações corrobora as principais diretrizes de competências (Bennett-Levy, 2006; Rodolfa et al., 2005; Roth \& Pilling, 2007). Outros autores destacam habilidades específicas como essenciais para o desenvolvimento de 
competências em TCC, por exemplo, a conceitualização cognitiva dos casos formulada de maneira colaborativa com o paciente (Neufeld \& Cavenage, 2010).

Para a aprendizagem das competências essenciais, Barletta, Fonseca e Nobre-Sandoval (2018) destacam a importância da reflexão acerca da prática terapêutica, incluindo a necessidade de sistematização da avaliação do ensino em TCC e da prática clínica. A adoção dessa conduta pode minimizar as lacunas entre a pesquisa baseada em evidências e a prática vigente. Apesar da necessidade de métodos precisos e eficientes de avaliar a competência da TCC, o progresso tem sido um tanto limitado em função da falta de unanimidade da área, devido à complexidade e às múltiplas dimensões inter-relacionadas para determinar as competências do terapeuta (Muse \& McManus, 2016). Entende-se que a dificuldade nesse processo perpassa por alguns aspectos, como: modelos e frameworks recentes de supervisão clínica em TCC (Bernard \& Goodyear, 2014), concepções equivocadas sobre a função da supervisão clínica (Falender, 2018), incentivo recente para a formação do supervisor clínico (Barletta, Fonseca, \& Nobre-Sandoval, 2018; Milne \& Reiser, 2016); falta de estabelecimento de metas educativas no treinamento e supervisão clínica (Corrie \& Lane, 2015). Outras questões a serem consideradas, além da própria avaliação em si, refere-se ao contexto na qual a supervisão e treinamento está inserida, incluindo as questões instrucionais, público-alvo e culturais de uma região.

Apesar dessa preocupação no cenário mundial, há uma lacuna e escassez de estudos sobre essa temática no Brasil (Honorato \& Barletta, 2016; Souza \& Padovani, 2015). Baseado nessa constatação e na breve contextualização sobre competências clínicas, este estudo visa realizar um levantamento no âmbito latino-americano sobre as competências necessárias para o terapeuta clínico da TCC.

\section{Método}

O presente estudo consiste em uma revisão integrativa da literatura (Mendes, Silveira, \& Galvão, 2008) em que, primeiramente, foi definida a questão de pesquisa: "quais são as competências necessárias a um terapeuta cognitivo-comportamental?". Foi realizado um levantamento bibliográfico em quatro bancos de dados, durante o mês de agosto de 2018: PsycINFO, Literatura Latino-americana e do Caribe em Ciências da Saúde (LILACS), Scientific Electronic Library Online (SciELO) e Periódicos Eletrônicos de Psicologia
(PePSIC). Os termos utilizados foram "competência/ competências" OR "habilidade/habilidades" AND "psicoterapia" OR "psicoterapeuta" OR "psicólogo clínico" e suas correspondências em inglês e espanhol. Apesar de a ênfase do presente estudo ser na TCC, objetivou-se buscar publicações sobre competências gerais, sem adicionar o termo "cognitivo-comportamental" justamente devido à escassez de estudos no âmbito nacional, incluindo em TCC. Além disso, sendo competências gerais (ou seja, que todo terapeuta precisa ter independentemente da abordagem), entende-se que o terapeuta em TCC também precisará dominar tais competências para entregar uma intervenção eficaz. Assim, os artigos precisavam contemplar competências do terapeuta na TCC, mesmo que concomitantemente com outras perspectivas teóricas, ou contemplar competências do terapeuta no geral, sem fazer referência a uma abordagem específica.

Foram incluídos nos resultados finais artigos de autores latino-americanos, escritos em português, inglês e espanhol, publicados nos últimos 12 anos, na área da Psicologia e que abordassem as competências do terapeuta como assunto principal. A escolha desse período de busca deu-se devido ao fato de que, após o movimento inicial da IAPT, intensificou-se a busca por uma formação baseada em competências, como supracitado. Além disso, como as diretrizes de Roth e Pilling (2007), tem sido ainda a mais utilizada atualmente, optou-se por buscar publicações posteriores, a fim de verificar se houve grandes mudanças no cenário.

Foram excluídas publicações que não fossem artigos, como capítulos de livros, resenhas e teses, que estivessem em outros idiomas diferentes dos supracitados, que abordassem as competências do terapeuta apenas em outras abordagens sem incluir a TCC, que tratassem competências do psicólogo em outros ambientes que não fossem clínicos e aqueles que tratavam apenas sobre competências do supervisor. Também foram excluídos artigos repetidos.

$\mathrm{Na}$ busca inicial, realizada pela primeira autora, foram encontradas 652 publicações, porém, após passar por todos os filtros dos critérios de inclusão e exclusão, resultaram 10 artigos que foram lidos na íntegra por duas pesquisadoras separadamente. Desse total, dois artigos foram excluídos por não contemplarem os critérios de inclusão e exclusão e oito artigos foram incluídos na amostra deste trabalho. O fluxograma da Figura 1 detalha esse processo.

Para a análise dos dados, foi construída uma folha de codificação contendo itens de informações 
relevantes ao objetivo da pesquisa, separados em: a) identificação do artigo e publicação, como título e autores; b) características do trabalho, como objetivo, participantes e procedimentos; c) definição de competência e d) identificação das competências. O objetivo dessa estratégia foi sistematizar os conteúdos encontrados nos artigos da revisão.

\section{Resultados}

Para organizar a seção de resultados, inicialmente serão descritas as características dos artigos e, em seguida, serão apresentadas as competências identificadas nos trabalhos. As categorias definidas para análise seguiram a literatura (Honorato \& Barletta, 2016), a saber, "analítica: conhecimento teórico sobre a TCC e seus princípios; instrumental: capacidade prática e de aplicação das técnicas e procedimentos em TCC; sociais: forma de se relacionar, de postura e de conduta na relação com o paciente; autoconhecimento do terapeuta: reflexão da própria atuação e das próprias emoções, eliciadas no contexto terapêutico" (p. 133).

\section{Características dos Artigos}

Apenas um dos artigos era de autores chilenos, enquanto todos os outros eram de autores brasileiros. Além disso, dois foram publicados nos últimos cinco anos, sendo considerados recentes. Em relação ao tipo de estudo, havia três teóricos, duas revisões sistemáticas de literatura e três pesquisas de campo. Quanto ao suporte teórico, quatro artigos eram especificamente de TCC, um abordava três teorias (centrada na pessoa, psicanálise e TCC) e outros três eram gerais. A Tabela 1 apresenta uma síntese das características dos artigos, incluindo o tipo de estudo, país, autores, ano, abordagem teórica e objetivos.

\section{Competências}

As competências analíticas descritas nos textos foram: a) conhecimento acerca do sistema de crenças

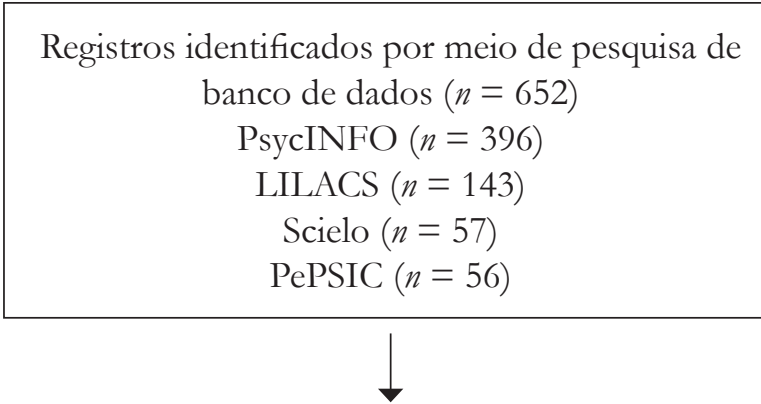

Registros selecionados $(n=442)$

$$
\begin{gathered}
\text { PsycINFO }(n=186) \\
\text { LILACS }(n=143) \\
\text { Scielo }(n=57) \\
\text { PePSIC }(n=56)
\end{gathered}
$$

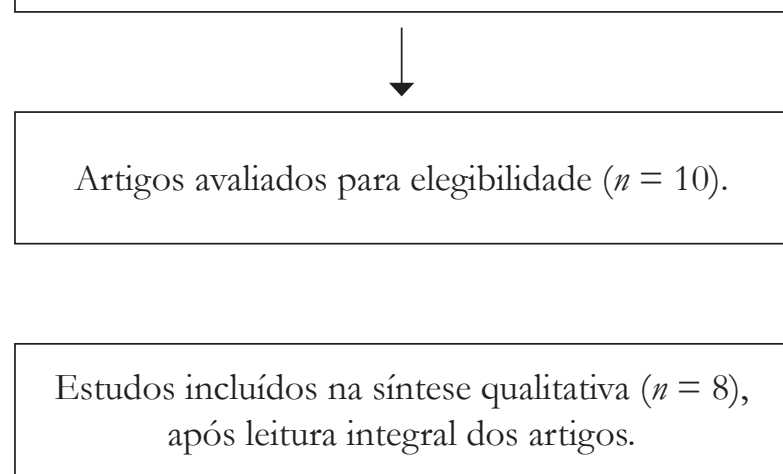

Figura 1. Fluxograma da revisão
Registros excluídos pelos filtros da base.

Critérios utilizados: apenas artigos, nos idiomas português, inglês e espanhol.

Artigos excluídos com base nos critérios: competências de outras abordagens (sem TCC), competências do psicólogo não clínico, competências do supervisor. 
religiosas do paciente (artigo 1), b) conhecimento acerca do modelo teórico da abordagem com a qual trabalha (artigos 4, 6,7) e c) conhecimento acerca dos princípios básicos das técnicas específicas a cada abordagem (artigos 6,7). Já com relação às competências sociais, as habilidades empáticas foram as mais citadas pelos artigos, seguidas pelas habilidades de comunicação, de assertividade e pelo respeito, conforme mostra a Tabela 2 .

Entre as competências instrumentais, foram elencadas 18 habilidades ou componentes diferentes, totalizando 28 menções. Elencar e focar em metas terapêuticas foi a habilidade mais citada (artigos 6, 7 e 8) entre as competências instrumentais. Aquelas citadas por dois artigos foram: proposição de planos de ação (artigos 5 e 6), conceitualização cognitiva, psicoeducação, técnicas de registro de pensamento, identificação e explicação de distorções cognitivas e planejamento da próxima sessão (artigos 6 e 7).

Outras competências instrumentais foram citadas apenas em um artigo. Essas competências foram: incentivar a autoexploração do paciente, usar técnicas

Tabela 1.

Sintese das Características dos Artigos

\begin{tabular}{|c|c|c|c|c|c|}
\hline Artigo & Tipo & País & Autores/Ano & Aborda-gem & Objetivo do artigo \\
\hline 1 & $\mathrm{~T}$ & Brasil & $\begin{array}{l}\text { Peres, Simão, \& } \\
\text { Nasello (2007) }\end{array}$ & Geral & $\begin{array}{l}\text { Discutir a integração das dimensões espirituais/ } \\
\text { religiosas na psicoterapia. }\end{array}$ \\
\hline 2 & $\mathrm{~T}$ & Chile & $\begin{array}{l}\text { Fernández, Mella, } \\
\text { Chenevard, García, } \\
\text { Cáceres, \& Vergara } \\
(2008)\end{array}$ & Geral & $\begin{array}{l}\text { Apresentar uma sistematização teórica das } \\
\text { variáveis não específicas (fatores comuns a } \\
\text { todas as abordagens) intervenientes no processo } \\
\text { psicoterapêutico (dentre elas, competências do } \\
\text { terapeuta). }\end{array}$ \\
\hline 3 & RS & Brasil & $\begin{array}{l}\text { Sartori, Del Prette, } \\
\text { \& Del Prette (2017) }\end{array}$ & Geral & $\begin{array}{l}\text { Esclarecer as características dos psicoterapeutas } \\
\text { favoráveis à formação e à manutenção da aliança, } \\
\text { assim como, avaliar habilidades sociais que a } \\
\text { literatura situa como parte desses requisitos. }\end{array}$ \\
\hline 4 & PC & Brasil & $\begin{array}{l}\text { Freitas \& Noronha } \\
(2007)\end{array}$ & $\begin{array}{l}\text { ACP, Psicaná- } \\
\text { lise e TCC }\end{array}$ & $\begin{array}{l}\text { Investigar as habilidades consideradas mais e } \\
\text { menos importantes para os alunos-terapeutas, } \\
\text { segundo os supervisores de diferentes } \\
\text { perspectivas teóricas. }\end{array}$ \\
\hline 5 & RS & Brasil & $\begin{array}{l}\text { Martins, Oliveira, } \\
\text { Vasconcelos, \& } \\
\text { Carvalho (2018) }\end{array}$ & TCC & $\begin{array}{l}\text { Traçar o panorama da produção em português } \\
\text { de trabalhos acadêmicos sobre empatia e relação } \\
\text { terapêutica, dando enfoque para a Terapia } \\
\text { Cognitivo-Comportamental. }\end{array}$ \\
\hline 6 & PC & Brasil & $\begin{array}{l}\text { Barletta, Delabrida, } \\
\text { \& Fonseca (2011) }\end{array}$ & TCC & $\begin{array}{l}\text { Descrever a percepção de } 15 \text { terapeutas iniciantes } \\
\text { no último ano de graduação sobre seu próprio } \\
\text { desenvolvimento em TCC. }\end{array}$ \\
\hline 7 & $\mathrm{~T}$ & Brasil & $\begin{array}{l}\text { Barletta, Fonseca, } \\
\text { \& Delabrida (2012) }\end{array}$ & TCC & $\begin{array}{l}\text { Discutir a importância da supervisão clínica como } \\
\text { instrumento essencial para o desenvolvimento } \\
\text { de competências terapêuticas em TCC em } \\
\text { graduandos de Psicologia. }\end{array}$ \\
\hline 8 & PC & Brasil & $\begin{array}{l}\text { Barletta, Fonseca, } \\
\text { \& Oliveira (2011) }\end{array}$ & TCC & $\begin{array}{l}\text { Avaliar o uso da transcrição do atendimento e da } \\
\text { observação em sala de espelho unilateral como } \\
\text { estratégias para o treinamento e desenvolvimento } \\
\text { de competência clínica em psicoterapia. }\end{array}$ \\
\hline
\end{tabular}

Nota. T - artigo teórico; RS - revisão sistemática; PC - pesquisa de campo; ACP - Abordagem Centrada na Pessoa. 
Tabela 2.

Competências Sociais

\begin{tabular}{|c|c|c|c|c|c|c|c|c|c|c|}
\hline \multirow{2}{*}{ Componentes } & \multicolumn{9}{|c|}{ Artigos } & \multirow[t]{2}{*}{ Total } \\
\hline & 1 & & 2 & 3 & 4 & 5 & 6 & 7 & 8 & \\
\hline Habilidades empáticas & $\mathrm{X}$ & & $\mathrm{X}$ & $\mathrm{X}$ & $\mathrm{X}$ & $\mathrm{X}$ & $\mathrm{X}$ & - & - & 6 \\
\hline Habilidades de comunicação & $x$ & & $\mathrm{X}$ & $\mathrm{X}$ & - & - & - & - & - & 3 \\
\hline Habilidades assertivas, direito e cidadania & - & & $\mathrm{X}$ & $\mathrm{X}$ & - & $\mathrm{X}$ & - & - & - & 3 \\
\hline Habilidades de expressão do sentimento positivo & - & & - & $\mathrm{X}$ & - & - & - & - & - & 1 \\
\hline $\begin{array}{l}\text { Ser sensível as questões socioculturais, socioeconômicas } \\
\text { e educacionais }\end{array}$ & - & & - & - & - & - & $\mathrm{X}$ & - & - & 1 \\
\hline Estabelecer e manter uma relação colaborativa & 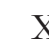 & & - & - & - & - & $\mathrm{X}$ & $\mathrm{X}$ & - & 3 \\
\hline Postura ética & $x$ & & $\mathrm{X}$ & - & $\mathrm{X}$ & - & - & - & $\mathrm{X}$ & 4 \\
\hline Estabelecer relação terapêutica & - & & $\mathrm{X}$ & - & - & $\mathrm{X}$ & - & - & - & 2 \\
\hline Respeito & $x$ & & $\mathrm{X}$ & - & - & - & $\mathrm{X}$ & - & - & 3 \\
\hline Não julgamento & - & & $\mathrm{X}$ & - & - & - & $\mathrm{X}$ & - & - & 2 \\
\hline Total & 5 & 8 & 4 & 2 & 3 & 5 & 1 & 1 & & 28 \\
\hline
\end{tabular}

de prevenção de recaídas, tratar clientes com múltiplos problemas segundo o formato de psicoterapia breve, aplicar técnicas de resolução de problemas, realizar a estruturação da sessão, tratar clientes com múltiplos problemas segundo o formato de psicoterapia breve, aplicar técnicas de resolução de problemas, realizar a estruturação da sessão, flexibilizar a sessão de acordo com as necessidades de cada paciente, realizar checagem de humor, estabelecer agenda da sessão, fazer resumos ao longo da sessão, fazer uso do feedback, eliciar e identificar pensamentos automáticos, utilizar atividades programadas e registro destas, realizar sessões de reforço, follow-up e término, diálogo socrático.

Finalmente, com relação às competências de autoconhecimento, três artigos mencionaram cinco diferentes componentes ou atitudes dos terapeutas. Tais componentes foram: a) o artigo 2 aponta o manejo das emoções e dificuldades do terapeuta ao longo da sessão, b) o artigo 6 destaca as diretrizes da (AADPRT), que inclui a educação continuada, a busca por supervisão adequada e estar aberto para observações das sessões psicoterápicas, seja ao vivo ou gravadas e c) o artigo 7 aponta a avaliação dos resultados da própria intervenção pelo terapeuta.

\section{Discussão}

O reduzido número de artigos encontrados nesta pesquisa corrobora a afirmação de que o estudo sobre competências do terapeuta no âmbito brasileiro e latino-americano ainda é incipiente (Alves, 2016; Barletta \& Neufeld, 2020; Honorato \& Barletta, 2016; Milne \& Reiser, 2016). Na presente revisão, foi encontrado apenas um artigo de autores não brasileiros, sugerindo uma escassez ainda maior nessa temática ao se falar de América Latina. Faz-se uma relação com a própria Psicologia na região, em que a Psicologia Clínica tem uma história tenra na América Latina, com maior difusão em dois países: Brasil e Argentina (Neufeld \& Carvalho, 2017). Somado a isso, essas autoras apontam a pouca ênfase inicial em pesquisa na região. Tais aspectos podem impactar na produção sobre a temática. Outro ponto percebido foi que três artigos são de um mesmo grupo de autores, o que levanta a um questionamento se o interesse dessa temática está apenas em poucos grupos de pesquisa e pesquisadores isolados, desfavorecendo a troca e disseminação desse conhecimento.

Além disso, nota-se que metade dos artigos encontrados possuem como aporte teórico a TCC. Como apontado anteriormente, segundo Barletta (2018), a busca por competências para a clínica se tornou mais forte após o movimento da psicologia baseada em evidências (APA, 2005). Ao se pensar na Psicologia Clínica, esse movimento ganhou força com a proposta da IAPT em identificar competências essenciais (Roth \& Pilling, 2007). Na contramão desse movimento, encontra-se uma lacuna de produção científica em âmbito mundial. Em justificativa, Falender 
(2018) ressalta que o olhar para a pesquisa sobre supervisão clínica tem aumentado apenas nos últimos anos devido a transformação sobre o seu entendimento, incluindo suas funções e sua importância. Ao ressignificar concepções consideradas inadequadas sobre a supervisão clínica que a acompanharam ao longo da história da formação do terapeuta, foi possível estabelecer a necessidade de pesquisa sobre a temática.

Essa escassez também foi encontrada em outros estudos. Por exemplo, Honorato e Barletta (2016), ao fazerem um levantamento sobre as competências do terapeuta cognitivo-comportamental, não encontraram nenhum estudo com delineamento experimental no Brasil. Esses autores relacionaram ter poucas pesquisas nessa área na América Latina, ou seja, que ainda é um momento de exploração inicial do tema nessa região, mas reforçam a importância de partir para pesquisas mais robustas.

Somado a isso, recentemente novas formas pedagógicas têm sido elencadas como mais eficazes, o que também impacta no treinamento de terapeutas e na supervisão clínica (Bacich \& Moran, 2018). Outro aspecto que vale ressaltar é a distância entre o profissional da prática clínica e o pesquisador, uma discussão já apresentada na literatura sobre a necessidade da pesquisa ultrapassar os muros da universidade para alcançar a comunidade (Reis \& Barbosa, 2018). Assim, a parceria entre pesquisador e psicólogos clínicos pode ser um avanço nas investigações na prática baseada em evidências.

Tratando-se do tipo de estudo, foram encontrados dois artigos de revisão sistemática. Entretanto, essas duas revisões abordaram apenas algumas dimensões de competências, não havendo a pretensão de mapear todas as competências necessárias ao terapeuta. O artigo 5 se propôs a realizar uma revisão sobre empatia e relação terapêutica, e o artigo 3 abordou as competências sociais voltadas para o estabelecimento da relação terapêutica. Esse movimento pode ser atribuído à complexidade e às múltiplas dimensões de competências do terapeuta proposto pelas diferentes diretrizes, como destacado por Muse e McManus (2016).

Entre as competências encontradas nos artigos que fizeram parte da amostra do presente estudo, observou-se que as sociais e instrumentais foram as mais citadas. Entende-se que a relação terapêutica é um elemento essencial em qualquer prática psicoterápica, independentemente da orientação teórica que a sustenta, sendo considerada como uma competência inespecífica ou geral (Kaplowitz, Safran, \& Muran,
2011; Roth \& Pilling, 2007). Ainda que haja especificidades da relação terapêutica de acordo com a orientação teórica, Newman (2013) aponta componentes comuns semelhantes a qualquer psicoterapia, fortalecendo a ideia de uma competência geral.

A literatura ainda reforça a importância do estabelecimento da relação terapêutica de forma adequada para que as intervenções específicas possam ser possíveis (Alves, 2016; Newman, 2013; Prasko, Vyskocilova, Slepecky, \& Novotny, 2012). Os achados da presente pesquisa mostraram estudos que citaram especificamente a relação terapêutica e outros que trouxeram componentes específicos do vínculo estabelecido na terapia e da relação interpessoal nesse contexto, como habilidades empáticas, de comunicação e assertivas.

A postura ética, outro componente citado nos resultados deste trabalho, está intimamente associada com a competência de desenvolvimento profissional, envolvendo fatores, como pensamento crítico, relações interpessoais, autoconhecimento e autocuidado (Wise, 2008). Barletta, Delabrida e Fonseca (2011) também apontam a importância das habilidades sociais para o estabelecimento da postura ética profissional, em especial, quando se trata da diversidade, incluindo diferenças sociais, culturais, econômicas e educacionais. Newman (2013) ressalta que a conquista da competência cultural e ética na TCC também é pertinente ao campo da psicoterapia como um todo, já que todos os profissionais de saúde mental enfrentam os desafios inerentes ao profissionalismo, à prática qualificada e ao bem-estar dos clientes à frente de vieses pessoais.

Os textos que mais indicaram competências instrumentais possuem como aporte teórico a TCC, que consiste em uma terapia estruturada, sistematizada e planejada, a aplicação de técnicas é de fundamental importância (Honorato \& Barletta, 2016), ainda que não seja uma competência exclusiva. Partindo desse entendimento, acredita-se que seria esperado que tais artigos dessem destaque para as competências instrumentais, ou pelo menos, citassem-nas.

$\mathrm{Na}$ literatura internacional, a flexibilidade da intervenção de acordo com as demandas do paciente é muito citada (Fung \& Lo, 2017), entretanto, nos resultados deste estudo foi citada apenas uma vez. Sabe-se que tal habilidade é essencial para que a intervenção seja eficaz para cada indivíduo e suas peculiaridades subjetivas, tornando a terapia dinâmica (Barletta, Fonseca, \& Delabrida, 2012). Honorato e Barletta (2016) também destacam que a habilidade do terapeuta em adaptar a estrutura da intervenção de acordo com as necessidades 
de cada paciente é fundamental, pois cada um possui peculiaridades. As autoras destacam que, na TCC, a manutenção da estrutura da terapia é importante, porém a flexibilização de acordo com a demanda, sem descaracterizá-la, garante o sucesso terapêutico.

Esse raciocínio levanta uma reflexão sobre a importância da relação terapêutica na TCC, que é duramente criticada por não dar valor a essa competência, como se atribuísse o sucesso terapêutico apenas às competências técnicas e específicas (Dalal, 2018). Esse aspecto reforça o entendimento anterior, em que as competências sociais são essenciais para o estabelecimento de intervenções específicas, levando em consideração as idiossincrasias de cada um (Newman, 2013; Prasko et al., 2012) e consequentemente, levando ao sucesso terapêutico. Ou seja, ainda que com diferenças e especificidades da relação terapêutica em diferentes teorias, essa relação é fundamental em qualquer processo psicoterápico, incluindo a TCC (Newman, 2013).

Outra competência descrita nos resultados foi a habilidade do terapeuta em realizar a formulação ou conceitualização do caso que, de acordo com Neufeld e Cavenage (2010), é um aspecto fundamental na TCC para compreender as necessidades do paciente e elaborar um plano terapêutico. As autoras também destacam que a formulação de caso é importante para que o terapeuta possa realizar a relação entre a teoria e o caso apresentado a ele, sistematizar a avaliação, intervenção e monitoramento do progresso do caso, sendo reconhecida como uma competência fundamental. Assim, destaca-se a importância das competências analíticas encontradas nos resultados da presente revisão, somada as outras já citadas.

Por exemplo, um dos artigos encontrados (Peres, Simão, \& Nasello, 2007) discorre sobre o conhecimento sobre o sistema de crenças religiosas e seu papel sobre a queixa e o funcionamento global do paciente. Estudos internacionais da área corroboram esse dado (Daniels \& Fitzpatrick, 2013), apontando que um terapeuta competente reconhece que as crenças do paciente (ou ausência de crenças) sobre a espiritualidade são fundamentais para sua visão de mundo e podem influenciar o funcionamento psicossocial. Para desenvolver uma conceitualizaçao eficiente, é necessário que o terapeuta faça uso das competências analíticas, instrumentais e sociais.

A última categoria refere-se ao autoconhecimento e à autorreflexão do terapeuta. Nota-se que os estudos trouxeram a importância de o terapeuta ter consciência dos próprios valores e crenças e como estes impactam no tratamento do paciente (Alves, 2016; Honorato \& Barletta, 2016; Muse \& McManus, 2016). Além do automonitoramento, é importante que o terapeuta tenha manejo adequado dessas questões ao longo da intervenção, para que elas não tenham um impacto negativo no tratamento. É importante ressaltar que tais competências são bastante enfatizadas nos estudos relacionados às diversidades culturais, espirituais e sexuais, destacando a importância de o terapeuta assumir uma postura de alteridade nesses casos, de forma ainda mais intensa que em outros (Daniels \& Fitzpatrick, 2013; Fung \& Lo, 2017).

O desenvolvimento profissional, treinamento, supervisão e educação continuada potencializam o desenvolvimento da competência autorreflexiva do terapeuta (Rodolfa et al., 2005), reforçando diferentes habilidades em um terapeuta pouco experiente até aquele com maior prática (Newman, 2013). Apesar de os estudos desta revisão não terem discutido essa competência especificamente, Barletta et al. (2011), bem como outras autoras internacionais (Muse \& McManus, 2016), apontaram a importância de o terapeuta recorrer à supervisão quando necessário.

Newman (2013) destaca que o autocuidado e a qualidade de vida melhorados são considerações importantes para aqueles que empreendem o trabalho desafiador de estar nas profissões de ajuda. A TCC pode estar no centro desses esforços para ajudar os terapeutas a se beneficiarem dos próprios métodos, tanto na melhora de própria saúde mental, quanto sendo modelos para seus pacientes. Os resultados da presente pesquisa ressaltaram a importância do bem-estar emocional do terapeuta (Fernández et al., 2008) e do uso da terapia pessoal (Freitas \& Noronha, 2007) como forma de cuidado, ainda que não fossem aspectos elencados como competência.

É interessante destacar que um dos estudos encontrados (Fernández et al., 2008) apontou outros fatores como intervenientes no sucesso da terapia. Por exemplo, variáveis do paciente, incluindo as características demográficas, diagnóstico, crenças e expectativas acerca da terapia e disposição pessoal, e variáveis do terapeuta, como atitudes, fatores de personalidade, competências, nível de experiência e bem-estar emocional. Todavia, baseado no que foi discutido neste estudo, pode-se dizer que, apesar de existirem fatores que fogem ao controle do terapeuta, a interferência negativa de destes para a mudança terapêutica pode ser atenuada pelo aprimoramento contínuo das competências (Barletta \& Neufeld, 2020; Bennett-Levy, 
2006; Muse \& McManus, 2016), entendendo que o uso de evidências na prática clínica é associado a melhores resultados terapêuticos.

\section{Considerações Finais}

A presente revisão objetivou contribuir com a temática competências do terapeuta da TCC, fazendo uma revisão com textos da região latino-americana. Este estudo procurou contribuir com os resultados referentes a estudos latino-americanos, dado que o estudo sobre esse tema é consideravelmente mais volumoso e mais robusto na América do Norte e na Europa. Entre os principais achados da presente revisão estão as competências essenciais descritas nos artigos, que incluíram as analíticas, instrumentais, sociais e autorreflexivas. Dentre estas, considera-se que a maior contribuição foi em relação às competências sociais, como uma competência inespecífica ou geral, necessária a todas as psicoterapias independentemente da orientação teórica de base. Uma vez que o foco da presente pesquisa passava pelas competências do terapeuta cognitivo-comportamental, traduziu-se a importância da relação terapêutica para a condução efetiva das especificidades da TCC. Ou seja, para que o protocolo específico, a estrutura e a técnica utilizada na TCC sejam efetivas e levem ao sucesso terapêutico, a relação interpessoal estabelecida adequada é fundamental. Esse entendimento vai contra as críticas à TCC que argumentam que essa teoria robotiza o processo terapêutico sem considerar as idiossincrasias da pessoa em atendimento.

Entre as dificuldades do estudo, ao fazer a busca, foi encontrado apenas um estudo fora do Brasil e sete estudos brasileiros. Nesse sentido, entende-se que a amostra que compôs o presente estudo pode ser considerada uma limitação que não permite traçar generalizações. Por outro lado, traz à tona a discussão sobre a importância de a pesquisa voltar seu olhar para o terapeuta latino-americano. Outra limitação do estudo reside no fato de que esta revisão consiste em um dos possíveis recortes do tema, sendo que, em futuras pesquisas, outros focos podem ser investigados. Um exemplo são as competências necessárias ao terapeuta que pratica abordagens de terceira onda das terapias cognitivas, como a Terapia de Aceitação e Compromisso, Terapia Cognitiva baseada em Mindfulness, entre outras.

Levando isso em consideração, bem como a própria dificuldade de sistematização das competências trazida pela literatura, sugere-se pesquisas que ampliem a busca com diferentes descritores, como "terapeuta(s) cognitivo(s)", por exemplo. Além disso, outras bases de dados podem ser consultadas. Outra possível estratégia seria a busca direta na produção científica pessoal dos autores latino-americanos encontrados, a fim de favorecer a recuperação de materiais importantes. Outra sugestão é a busca de teses e dissertações que podem não ter tido seus trabalhos publicados.

A partir desse compilado, acredita-se que uma agenda de pesquisa possa dar prioridade para estudos empíricos, visando fortalecer o movimento da psicologia baseada em evidências. Por exemplo, pesquisas de avaliação de competências a partir de observação de gravações de sessão, com a utilização de instrumentos com evidências de validade, bem como pesquisas que avaliem os programas de treinamento de terapeutas a partir do resultado da intervenção.

\section{Referências}

Allan, R., McLuckie, A., \& Hoffecker, L. (2016). Clinical Supervision of Psychotherapists: A Systematic Review. The Campbell Collaboration, 5(2), 1-14. doi: 10.1300/J010v32n04_01

Alves, S. D. C. O. (2016). Competências do terapeuta cognitivo-comportamental. Revista Psicologia em Foco, 8(12), 51-66. Recuperado de http://www. revistas.fw.uri.br/index.php/psicologiaemfoco/ article/viewFile/2465/2179

American Psychological Association (2005). Report of the 2005 presidential task force on evidencebased practice. Washington, DC: Author. doi: 10.1037/0003-066X.61.4.271

Andersson, G. (2016). Internet-delivered psychological treatments. Annual Review of Clinical Psychology, 12, 157-179. doi: 10.1146/ annurev-clinpsy-021815-093006

Bacich, L., \& Moran, J. (2018). Metodologias ativas para uma educação inovadora: Uma abordagem teórico-prática. São Paulo: Editora Penso.

Barletta, J. B., \& Neufeld, C. B. (2020). Novos Rumos da Supervisão Clínica em Terapia Cognitivo-Comportamental: conceitos, modelos e estratégias baseadas em evidências. In: Neufeld, C. B., Falcone, E. M O., \& Rangé B. (orgs.), Procognitiva Programa de Atualização em TCC: ciclo 7 (pp. 9-49). Porto Alegre: Artmed.

Barletta, J. B., Delabrida, Z. N. C., \& da Fonsêca, A. L. B. (2011). Conhecimento, habilidades e atitude 
em TCC: Percepção de terapeutas iniciantes. Revista Brasileira de Terapias Cognitivas, 7(1), 21-29. doi: 10.5935/1808-5687.20110005

Barletta, J. B., Fonseca, A. L. B. D., \& Delabrida, Z. N. C. (2012). A importância da supervisão de estágio clínico para o desenvolvimento de competências em terapia cognitivo-comportamental. Psicologia: teoria e prática, 14(3), 153-167. Recuperado de http://pepsic.bvsalud.org/pdf/ptp/v14n3/ v14n3a13.pdf

Barletta, J. B., Fonseca, A. L. B., \& Nobre-Sandoval, L. A. (2018). Estratégias de ensino e de aprendizagem para a formação de terapeutas cognitivo-comportamentais. Em N. G. Araújo, J. P. Rubino \& M. I. S. Oliveira. (Eds.). Avaliação e intervenção na clínica em terapia cognitivo-comportamental: A prática ilustrada (pp. 460-482). Novo Hamburgo: Sinopsys.

Bennett-Levy, J. (2006). Therapist skills: A cognitive model of their acquisition and refinement. Behavioural and Cognitive Psychotherapy, 34(1), 57-78. doi: $10.1017 /$ S1352465805002420

Bernard, J. M., \& Goodyear, R. K. (2014). Fundamentals of clinical supervision (5th ed.). Upper Saddle River: Pearson.

Brandão, H. P., \& Bahry, C. P. (2014). Gestão por competências: Métodos e técnicas para mapeamento de competências. Revista do Serviço Público, 56(2), 179-194. doi: 10.21874/rsp.v56i2.224

Carbone, P. P., Tonet, H. C., Bruno, J. S. R., Silva, B. E., \& Izidio, K. (2016). Gestão por competências. Rio de Janeiro: Editora FGV.

Corrie, S., \& Lane, D. A. (2015). CBT supervision. Thousand Oaks: Sage.

Dalal, F. (2018). CBT: The Cognitive Behavioural Tsunami: Managerialism, Politics and the Corruptions of Science. Abingdon: Routledge.

Daniels, M. C., \& Fitzpatrick, M. (2013). Integrating spirituality in counselling and psychotherapy: Theoretical and clinical perspectives. Canadian Journal of Counselling and Psychotherapy, 47(3), 315-341. Recuperado de https://cjc-rcc.ucalgary.ca/article/ download/60807/46151

Falender, C. A. (2018). Clinical supervision - The missing ingredient. American Psychologist, 73(9), 1240-1250. doi: 10.1037/amp0000385
Fernández, P. M. S., Mella, M. F. R., Chenevard, C. L., García, A. E. E., Cáceres, D. I. E., \& Vergara, P. A. M. (2008). Variables Inespecíficas en Psicoterapia. Terapia Psicológica, 26(1), 89-98. doi: 10.4067/ S0718-48082008000100008

Freitas, F. A. D., \& Noronha, A. P. P. (2007). Habilidades do psicoterapeuta segundo supervisores: Diferentes perspectivas. Psic: revista da Vetor Editora, 8(2), 159-166. Recuperado de http://pepsic. bvsalud.org/scielo.php?script=sci_arttext\&pi$\mathrm{d}=$ S167673142007000200006

Fung, K., \& Lo, T. (2017). An integrative clinical approach to cultural competent psychotherapy. Journal of Contemporary Psychotherapy, 47(2), 65-73. doi: 10.1007/s10879-016-9341-8

Honorato, A. R. R., \& Barletta, J. B. (2016). Estratégias de ensino e competências desenvolvidas na supervisão clínica em Terapia Cognitivo-Comportamental: uma revisão integrativa da produção nacional. Em A. L. B Fonseca, M. S. S. Mariano \& J. B. Barletta (Eds.). Comportamento, Desenvolvimento e Cultura: análise de contexto. (pp. 125-147). Curitiba: Editora CRV.

Kaplowitz, M. J., Safran, J. D., \& Muran, C. J. (2011). Impact of therapist emotional intelligence on psychotherapy. Journal of Nervous and Mental Disease, 199(2), 74-84. doi: 10.1097/ NMD.0b013e3182083efb

Martins, J. S., Oliveira, L. S., Vasconcelos, R. C. D. C., \& Carvalho, A. L. N. (2018). Empatia e relação terapêutica na psicoterapia cognitiva: Uma revisão sistemática. Revista Brasileira de Terapias Cognitivas, 14(1), 50-56. doi: 10.5935/1808-5687.20180007

Melnik, T., Meyer, S. B., \& Sampaio, M. I. C. (2019). Relato de experiência docente: A primeira disciplina no Brasil sobre a Prática da Psicologia Baseada em Evidências ministrada no Instituto de Psicologia da Universidade de São Paulo. Psicologia: Teoria e Pesquisa, 35, 1-5. doi: 10.1590/0102.3772e35418

Mendes, K. D. S., Silveira, R. C. C. P., \& Galvão, C. M. (2008). Revisão Integrativa: Método de pesquisa para a incorporação de evidências na saúde e na enfermagem. Texto Contexto Enferm, 17(4), 758-64. Recuperado de https://www.redalyc.org/ pdf/714/71411240017.pdf

Milne, D. L. (2018). Evidence-based CBT supervision: Principles and practice. $2^{\text {nd }}$ edition. Hoboken, NJ: Wiley.

Psico-USF, Bragança Paulista, v. 26, n. 1, p. 141-152, jan./ mar. 2021 
Milne, D. L., \& Reiser, R. (2016). Supporting our supervisors: Sending out an SOS. The Cognitive Behaviour Therapist, 9(19), 1-12. doi: 10.1017/ S1754470X15000616

Muse, K., \& McManus, F. (2016). Expert insight into the assessment of competence in cognitive-behavioural therapy: A qualitative exploration of experts' experiences, opinions and recommendations. Clinical psychology \& psychotherapy, 23(3), 246-259. doi: 10.1002/cpp.1952

Neufeld, C. B., \& Cavenage, C. C. (2010). Conceitualização cognitiva de caso: Uma proposta de sistematização a partir da prática clínica e da formação de terapeutas cognitivo-comportamentais. Revista Brasileira de Terapias Cognitivas, 6(2), 3-36. doi: 10.5935/1808-5687.20100014

Neufeld, C. B., \& de Carvalho, A. V. (2017). Latin America. Em S. G. Hofmann (Eds.). International Perspectives on Psychotherapy (pp. 121-137). New York: Springer.

Newman, C. F. (2013). Core competencies in cognitive-behavioral therapy: Becoming a bighly effective and competent cognitive-behavioral therapist. Abingdon: Routledge.

Newman, C. F., \& Kaplan, D. A. (2016). Supervision Essentials for Cognitive-Behavioral Therapy. Washington: American Psychological Association.

Pascual-Leone, A., Andreescu, C. A., \& Yeryomenko, N. (2015). Training novice psychotherapists: Comparing undergraduate and graduate students' outcomes. Counselling \& Psychotherapy Research, 15(2), 137-146. Recuperado de https://onlinelibrary.wiley.com/doi/pdf/10.1002/capr.12007

Peres, J. F. P., Simão, M. J. P., \& Nasello, A. G. (2007). Espiritualidade, religiosidade e psicoterapia. Revista de Psiquiatria Clinica, 34(1), 136-145. Recuperado de https://www.revistas.usp.br/acp/article/ download/17129/19132

Prasko, J., Vyskocilova, J., Slepecky, M., \& Novotny, M. (2012). Principles of supervision in cognitive behavioural therapy. Biomedical Papers, 156(1),70-79. doi: $10.5507 /$ bp.2011.022

Price, S. D., Callahan, J. L., \& Cox, R. J. (2017). Psychometric investigation of competency benchmarks. Training and Education in Professional Psychology, 11(3), 128-139. doi: $10.1037 /$ tep0000133

Psico-USF, Bragança Paulista, v. 26, n. 1, p. 141-152, jan./ mar. 2021
Rangé, B., Pavan-Cândido, C., \& Neufeld, C. B. (2017). Breve histórico das terapias em grupo e da TCCG. Em C. B. Neufeld \& B. Rangé (Eds.). Terapia Cogntivo-Comprtamental em Grupos: Das evidências à prática. (pp. 13-29). Porto Alegre: Artmed.

Reis, G. A., \& Barbosa, A. J. G. (2018). Formação de terapeutas cognitivo-comportamentais: Um estudo sobre o estado da arte. Revista Brasileira de Terapia Comportamental e Cognitiva, 20(1), 72-85. Recuperado de www.usp.br/rbtcc/index.php/RBTCC/article/ download/1138/555

Rodolfa, E., Bent, R., Eisman, E., Nelson, P., Rehm, L., \& Ritchie, P. (2005). A cube model for competency development: Implications for psychology educators and regulators. Professional Psychology: Research and Practice, 36(4), 347. doi: 10.1037\%2F0735-7028.36.4.347

Roth, A. D., \& Pilling, S. (2007). The competences required to deliver effective cognitive and behavioural therapy for people with depression and with anxiety disorders. Sub-Department of Clinical Health Psychology, University College London. Recuperado de http://webarchive.nationalarchives. gov.uk/20081106113436/http://www.dh.gov. uk/en/Publicationsandstatistics/Publications/ PublicationsPolicyAndGuidance/DH_078537

Sartori, R. M., Del Prette, A., \& Del Prette, Z. A. P. (2017). Habilidades Sociais do terapeuta na formação da aliança psicoterapêutica: Estudo de revisão. Revista Brasileira de Psicoterapia, 19(2), 29-43. Recuperado de https://s3-sa-east-1.amazonaws. com/publisher.gn1.com.br/rbp.celg.org.br/pdf/ v19n2a04.pdf

Souza, C. R. D., \& Padovani, R. D. C. (2015). Supervision in Cognitive Behavioral Therapy: Tracing other ways besides the school service. Psico-USF, 20(3), 461-470. doi: 10.1590/1413-82712015200308

Wenzel, A. (2018). Inovações em Terapia Cognitivo-Comportamental: Intervenções estratégicas para uma prática criativa. Artmed Editora.

Wise, E. (2008). Competence and scope of practice: Ethics and professional development. Journal of Clinical Psychology, 64(5), 626-637. doi: 10.1002/ jclp.20479

Recebido em: 24/10/2018

Reformulado em: 22/03/2020

Aprovado em: 13/05/2020 
Sobre as autoras:

Isabela Lamante Scotton é Mestranda em Psicologia pela Universidade de São Paulo (Laboratório de Pesquisa e Intervenção Cognitivo-Comportamental - LaPICC-USP).

ORCID: https://orcid.org/0000-0001-6757-7208

E-mail: isabela.scotton@gmail.com

Janaína Bianca Barletta é Pós-doutoranda em Psicologia pela Universidade de São Paulo (USP-RP), bolsista CNPqPDJ e pesquisadora do Laboratório de Pesquisa e Intervenção Cognitivo-Comportamental (LaPICC-USP).

ORCID: https://orcid.org/0000-0002-0834-4559

E-mail: janabianca@gmail.com

Carmem Beatriz Neufeld é Livre Docente pela FFCLRP-USP. Pós-Doutora em Psicologia pela UFRJ. Doutora e Mestre em Psicologia pela PUCRS. Coordenadora do Laboratório de Pesquisa e Intervenção Cognitivo-Comportamental - LaPICC-USP. Professora Associada do Departamento de Psicologia da FFCLRP-USP. Presidente da Federação Latino Americana de Psicoterapias Cognitivas e Comportamentais - ALAPCCO.

ORCID: https://orcid.org/0000-0003-1097-2973

E-mail: cbneufeld@usp.com

Contato com as autoras:

Isabela L. Scotton

Av. Bandeirantes, 3900

Ribeirão Preto-SP, Brasil

Telefones: (16) 3315-0197/(16) 98156-3344 